\title{
The Influence of the Capping Agent on the Oxidation of Silver Nanoparticles: Nano-impacts vs Stripping Voltammetry
}

\author{
Her Shuang Toh, ${ }^{[a]}$ Kerstin Jurkschat, ${ }^{[b]}$ and Richard G. Compton ${ }^{\star[a]}$
}

\begin{abstract}
The influence of capping agents on the oxidation of silver nanoparticles is studied using the electrochemical techniques of anodic stripping voltammetry and anodic particle coulometry ('nanoimpacts'). Five spherical silver nanoparticles each with a different capping agent (branched polyethylenimine (BPEI), citrate, lipoic acid polyethylene glycol (PEG) and polyvinylpyrrolidone (PVP)) are used to perform comparative experiments. In all cases, regardless of the capping agent, complete oxidation of the single nanoparticles was seen in anodic particle coulometry. The successful quantitative detection of the silver nanoparticle size displays the potential application of anodic particle coulometry for nanoparticle characterisation. In contrast, for anodic stripping voltammetry using nanoparticles drop casting, it is observed that the capping agent has a very significant effect on the extent of silver oxidation. All five samples gave a low oxidative charge corresponding to partial oxidation. It is concluded that the use of anodic stripping voltammetry to quantify nanoparticles is unreliable and this is attributed to nanoparticle aggregation.
\end{abstract}

\section{Introduction}

Scientific research has placed much emphasis on nanoparticles as their special properties are appealing towards many commercial applications. ${ }^{[1]}$ Due to the decrease in size ${ }^{1}$, nanoparticles tend to have a higher surface energy compared to bulk material, which may lead to instability. $\left.{ }^{[1 b}, 3\right]$ Hence, nanoparticles often require capping agents to aid their stabilisation. ${ }^{[4]}$ Capping agents work through various mechanisms which can be broadly categorised into five different types: electrostatic stabilisation, steric stabilisation, stabilisation by hydration forces, depletion stabilisation and stabilisation by van de Waals forces. ${ }^{[4]}$ Occasionally, multiple mechanisms can occur with certain capping agents, for example, in the case of branched polyethylenimine (BPEI) where it stabilises silver nanoparticles both electrostatically and sterically. ${ }^{[5]}$

The choice of capping agents on nanoparticles is crucial as the properties (such as size, shape and the interaction with solvent surroundings) of the nanoparticle are strongly influenced by it. ${ }^{[6]}$ For example, the polymeric chain length of polyethylene

[a] H. S. Toh, Prof R. G. Compton

Physical \& Theoretical Chemistry Laboratory

Oxford University

South Parks Road, Oxford, OX1 3QZ, United Kingdom

Email: Richard.Compton@chem.ox.ac.uk

[b] Dr. K. Jurkschat

Department of Materials

Oxford University

Begbroke Science Park

Sandy Lane, Yarnton, Oxford, OX5 1PF, United Kingdom

\footnotetext{
${ }^{1}$ A nanomaterial is defined by IUPAC as any material with one
} dimension smaller than $100 \mathrm{~nm} \cdot{ }^{\text {[2] }}$ glycol (PEG) is important in silver nanoparticle synthesis as the number of monomers in PEG affects the nanoparticle size. ${ }^{[7]}$ Apart from their main role of stabilising nanoparticles, some capping agents (i.e. citrate) are capable of the additional role of reducing metal ions (e.g. silver ions reduced by citrate ions) into metal nanoparticles. ${ }^{[8]}$ Therefore, the choice of capping agent is vital in the nanoparticle synthesis process.

Apart from their strong influence in the synthesis process, capping agents also affect nanoparticle properties. ${ }^{[9]}$ For example, gold nanoparticles with organic capping agents with changed side chains (alkyl, aromatic or cholesterol groups) display different interactions with gelatin solutions, forming composites of contrasting morphologies. ${ }^{[9 a]}$ In another example reported by Kuhn et al, it was demonstrated that with capping agents containing amine groups on platinum nanoparticles, the catalytic properties of the nano-platinum was poisoned, leading to a lower efficiency for ethylene hydrogenation and carbon monoxide oxidation. ${ }^{[9 b]}$ Otherwise, having a capping agent on a nanoparticle surface can promote certain properties. In an antibacterial study of silver nanoparticles, the usage of any of the three capping agents (Tween-80, sodium dodecyl sulphate (SDS) or polyvinylpyrrolidone (PVP)) resulted in an enhanced anti-bacterial activity. ${ }^{[9 \mathrm{c}]}$

Given the significant influence of capping agents on nanoparticle properties, the question arises as to whether capping agents affect the techniques used for nanoparticle detection and quantification. ${ }^{[10]}$ Optical methods such as ultraviolet-visible (UV-vis) spectrometry and Fourier transform infrared (FTIR) spectroscopy are often used to characterise nanoparticles. ${ }^{[11]}$ For UV-vis spectroscopy, spherical silver nanoparticles provide a characteristic maximum absorbance around $400 \mathrm{~nm}$ whilst spherical gold nanoparticles have a strong absorbance around $500 \mathrm{~nm} .^{[11 \mathrm{a}-\mathrm{c}]}$ Studies have shown that by varying the concentration of capping agents (polyvinyl alcohol (PVA) or L-glutamic acid) on silver nanoparticles, the UV-vis absorbance signal shifts and changes shape. ${ }^{[12]}$ Similarly, literature has reported that the UV-vis and FTIR signals of cadmium telluride (CdTe) semi-conductor nanocrystals change with the capping agent on their surface..$^{[11 d]}$ Thus, CdTe nanocrystals capped by mercaptoacetic acid, mercaptopropionic acid or 2-mercaptoethanol show different responses for the optical methods. Hence, the influence from the capping agents may result in possible scenarios where the data obtained may be misleading. For example, the possible scenario of oversizing the nanoparticles when a blueshift in UV-vis signal could be due to a concentration change in capping agent instead of a change in nanoparticle size.

In the present work, the electrochemical techniques of anodic stripping voltammetry and anodic particle coulometry (nano-impacts) are studied to determine if there is any influence from the capping agents on the response of silver nanoparticles. Anodic stripping voltammetry is a well-studied technique which is traditionally used to determine the concentration of metals 
ions in solution by pre - concentrating and oxidising them on a suitable electrode. ${ }^{[13]}$ It gives an improved sensitivity compared to cyclic voltammetry used to reduce the metallic ions because of its pre-concentration step. ${ }^{[13]}$ Recently, it has also been adapted to detect nanoparticles. ${ }^{[14]}$ One of the most commonly used techniques for pre-concentrating nanoparticle is drop casting, where an analyte solution is deposited onto the working electrode, the solvent is evaporated and a layer of analyte is left behind on the electrode. ${ }^{[15]}$ A previous study has reported that this method causes silver nanoparticles to aggregate ${ }^{2}$ on the electrode surface because of solvent evaporation. ${ }^{[14 a]}$ As increasingly aggregated nanoparticles are found on the surface, the percentage oxidation of the cast material in anodic stripping voltammetry decreases, leading to the phenomenon of partial oxidation ("incomplete stripping"). ${ }^{[17]}$ Since capping agents play a crucial role in nanoparticle stabilisation and their aggregation kinetics, it is plausible to speculate that they might have a significant effect on the aggregation caused by drop casting and hence on the signal obtained in anodic stripping voltammetry. ${ }^{[5]}$ Therefore, to determine if the capping agents may have influence on the electrochemical behaviour of silver nanoparticle aggregates, anodic stripping voltammetry are performed on five spherical, similar sized silver nanoparticles samples each with a different capping agent (BPEI, citrate, lipoic acid, PEG or PVP) as shown in Figure 1.

To further investigate the partial oxidation of nanoparticle aggregates, a study of single nanoparticle oxidation is essential. Therefore, anodic particle coulometry pursued as a method wellsuited for this purpose. It records the electrochemical signals of single nanoparticles impacting the electrode surface under Brownian motion in solution. ${ }^{[18]}$ As the silver nanoparticle hits the electrode surface held at a suitable oxidising potential, it is oxidised into silver(I) ions and this generates a current 'spike' which appears in a chronoamperogram. In turn, the charge<smiles>CCCNCCN(CCN)CCN(CCNCCN)CCN(CCNC)CCN(CCN)CCN</smiles><smiles>O=C(O)CCCCC1CCSS1</smiles>

Branched Polyethylenimine (BPEI)

Polyethylene Glycol (PEG)
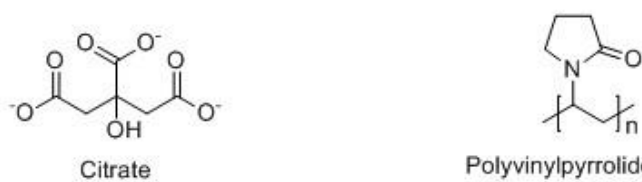

Polyvinylpyrrolidone (PVP)

Figure 1. The chemical structures of the capping agents studied.

\footnotetext{
${ }^{2}$ Following the IUPAC definition, the terms agglomeration and aggregation are defined as the reversible or irreversible adhesion of particles, respectively. ${ }^{[16]}$ In this context, the term aggregation will be used to refer to both aggregation and aggolomeration.
}

passed under the 'spike' can be related to the size of the nanoparticles via Faraday's first law and hence the effects of capping agents on the extent of partial oxidation of a single nanoparticle can be studied. To the best of our knowledge, anodic particle coulometry has only been performed on silver nanoparticles capped with citrate. ${ }^{[18 a, 18 c, 19]}$ Therefore, this work is the first to report on the influence of capping agents (i.e. BPEI, citrate, lipoic acid, PEG and PVP) on silver nanoparticles using anodic particle coulometry.

\section{Results and Discussion}

Five different samples of commercially available silver nanoparticles with various capping agents (i.e. branched polyethylenimine (BPEI) citrate, lipoic acid, polyethylene glycol (PEG) and polyvinylpyrrolidone(PVP)) were studied via anodic stripping voltammetry and anodic particle coulometry.

Silver nanoparticle suspensions of $100 \mathrm{pM}$ were drop cast onto a glassy carbon electrode and the modified electrode was used for anodic stripping voltammetry. It is found that the silver oxidation signal differs in size with the variance of capping agent. Next, the five silver samples were used for anodic particle coulometry and it is observed that the 'spikes' obtained (sizes inferred from the measured current) is quantitatively constant with the TEM characterisation data. Comparing the data from the two electrochemical techniques, it is deduced that the capping agent induces the partial oxidation of the silver nanoparticle in anodic stripping voltammetry; the latter are likely aggregated. ${ }^{[14 a]}$

\section{Anodic stripping voltammetry}

Silver nanoparticle modified glassy carbon electrodes prepared as described in the experimental section were used to perform anodic stripping voltammetry. All electrodes were modified with the same amount of silver. The electrochemical experiments were performed with five samples of commercially available silver nanoparticles each containing a different capping agent (BPEI, citrate, lipoic acid, PEG or PVP). Each experiment was repeated 3 times to ensure reproducibility. The voltammetric scan started oxidatively from $-0.6 \mathrm{~V}$ vs MSE in a $20 \mathrm{mM}$ sodium nitrate solution at a scan rate of $50 \mathrm{mV} \mathrm{s}^{-1}$ and the resulting voltammograms are summarised in Figure 2. A broad oxidation signal with a peak potential around $+0.05 \mathrm{~V}$ vs MSE was observed for all five samples. In the light of previous literature, it is assigned to the oxidation of metallic silver to silver $(\mathrm{I})$ ions ( $\mathrm{Ag}$ - $\left.\mathrm{e}^{-} \rightarrow \mathrm{Ag}^{+}\right) .^{[14 \mathrm{a}, 20]}$ The five scans show an oxidation signal which varies greatly in size. A slight difference in onset of the oxidation signal is likely due to the influence of the capping agent on the nanoparticle surface. The silver content of the $100 \mathrm{pM}$ nanoparticle suspension used to modify the glassy carbon electrode was $0.099 \mathrm{mg} \mathrm{mL}^{-1}$. Through Faraday's first law, it is expected that the oxidative charge from anodic stripping voltammery is $26 \mu \mathrm{C}$. As the oxidative charge under the signal from Figure 2 is recorded and plotted against the expected 


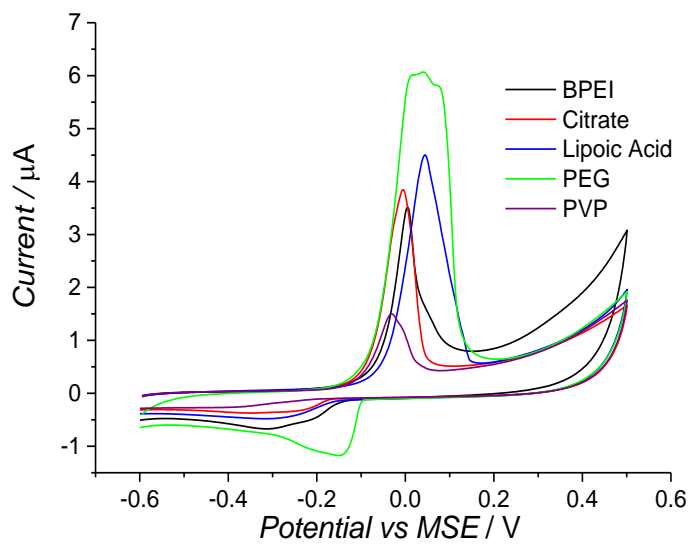

Figure 2. The oxidation of silver nanoparticles with different capping agents on a glassy carbon electrode in $20 \mathrm{mM}$ sodium nitrate at a scan rate of $50 \mathrm{mV} \mathrm{s}^{-1}$. Black: Branched Polyethylenimine (BPEI) capped silver nanoparticle; Red: Citrate capped silver nanoparticle; Blue: Lipoic acid capped silver nanoparticle; Green: Polyethylene Glycol (PEG) capped silver nanoparticle; Purple: Polyvinylpyrrolidone (PVP) capped silver nanoparticle.

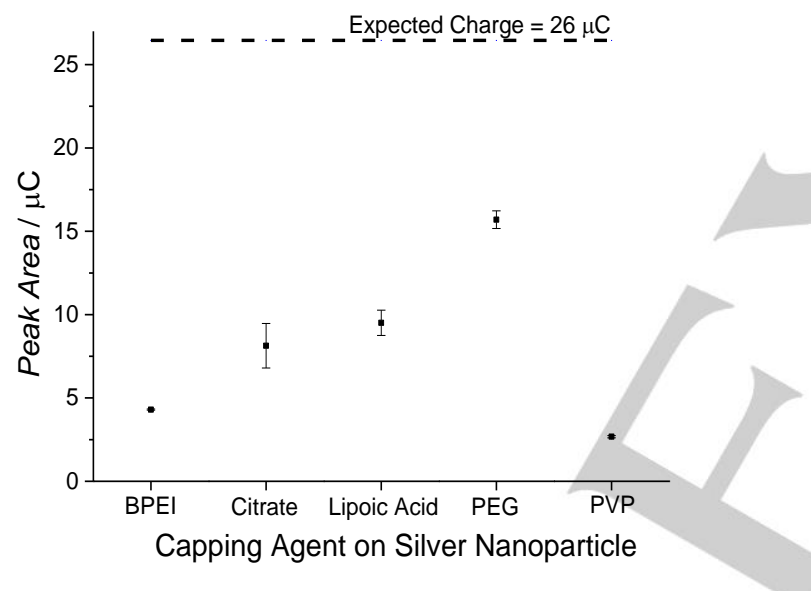

Figure 3. The oxidative charge under the peak area as the silver nanoparticles with different capping agents are oxidised on a glassy carbon electrode in 20 $\mathrm{mM}$ sodium nitrate at a scan rate of $50 \mathrm{mV} \mathrm{s}^{-1}$. The black dashed line indicates the amount of charge expected.

charge, it is observed that for all five types of the nanoparticles, slightly less than $100 \%$ oxidation occurred, as depicted in Figure 3 . It is also seen that the capping agents on the nanoparticle surface influence the size and height of the oxidation signal. In Figure 3, PEG capped silver nanoparticles are seen to give the oxidative signal of the largest magnitude (59\% oxidation) while PVP capped silver nanoparticles gave the lowest signal among the five (10\% oxidation). BPEl capped silver nanoparticles had $16 \%$ oxidation while citrate capped silver nanoparticles and lipoic acid capped silver nanoparticles gave $31 \%$ and $36 \%$ oxidation respectively.

For these anodic stripping voltammetry experiments, the working electrode was prepared through the method of drop casting. Literature reported that drop casting nanoparticles on the electrode surface causes aggregation to occur. ${ }^{[14 a]}$ It was also reported that the aggregated nanoparticles are stripped incompletely through anodic stripping voltammetry. ${ }^{[17]}$ Hence, it is reasonable to propose that in Figure 3 , there is an incomplete stripping of the silver nanoparticles. To compare the oxidation of nanoparticle aggregates against single nanoparticles, further investigation of the electrochemical behaviour of single nanoparticles is carried out through anodic particle coulometry in the next section.

\subsection{Anodic particle coulometry}

For anodic particle coulometry experiments, chronoamperograms of fifty seconds duration were recorded at $+0.6 \mathrm{~V}$ vs MSE. To ensure that a blank scan (Figure 4 black line) was obtained before any experiment with nanoparticles was performed, a current-time transient was measured on a carbon microdisc electrode placed in a $20 \mathrm{mM}$ sodium nitrate solution. This ensures that no contamination of nanoparticles arises from previous experiments. A typical blank is shown as the black line in Figure 4. The black line has been shifted upwards by $33 \mathrm{pA}$ vertically in Figure 4 for clarity. The red line in Figure 4 shows a typical scan that depicts single nanoparticles hitting the electrode through the observation of 'spikes' in the current-time transient held at $+0.6 \mathrm{~V}$ vs MSE. In this work, the 'spike' is the increase in current caused by the quantitative oxidation of the silver nanoparticle as it diffuses and hits the working electrode held at the oxidising potential. ${ }^{[18 a, 18 b]}$ In Figure 4 inset, multiple 'spikes' can be seen occurring over a time scale of milliseconds. When the chronoamperometric scan was held at $0 \mathrm{~V}$ vs MSE in the presence of silver nanoparticles, no 'spikes' were observed. Thus, the spikes are only observed when the potential held at the electrode is beyond the onset potential of silver oxidation, allowing us to attribute the 'spikes' to the oxidation of the silver nanoparticles impacting the electrode. ${ }^{[18 a, 18 b]}$ The blank scans carried out before experiment at $+0.6 \mathrm{~V}$ vs MSE also ensured that the 'spikes' observed in the chronoamperometry scan are caused by the nanoparticles added to the aliquot instead of any possible contamination.

The charge passed under each 'spike' can be used to calculate the nanoparticle radius through the equation below: [18a]

$$
R_{N P}=\sqrt[3]{\frac{3 Q A_{r}}{4 \pi F \rho}}
$$

where $R_{N P}$ is the nanoparticle radius, $Q$ is the total charge passed under a single 'spike', $A_{r}$ is the atomic molecular mass of silver of $107.9 \mathrm{~g} \mathrm{~mol}^{-1}, \mathrm{~F}$ is the Faraday constant and $\rho$ is the density of silver of $10.5 \times 10^{6} \mathrm{~g} \mathrm{~m}^{-3}$. The 'spikes' recorded can be converted into a histogram which depicts the size distribution of the nanoparticle sample. ${ }^{[18 a, 18 b]}$ For each of the five nanoparticle samples, multiple current-time transients were recorded till about 500 'spikes' were counted. Then, the data were processed to give a size distribution of the nanoparticle radii depicted in Figure 5 . The average radius of the five samples measured by anodic particle coulometry and TEM are tabulated in Table 1. The size of the nanoparticle obtained by TEM is not affected by 


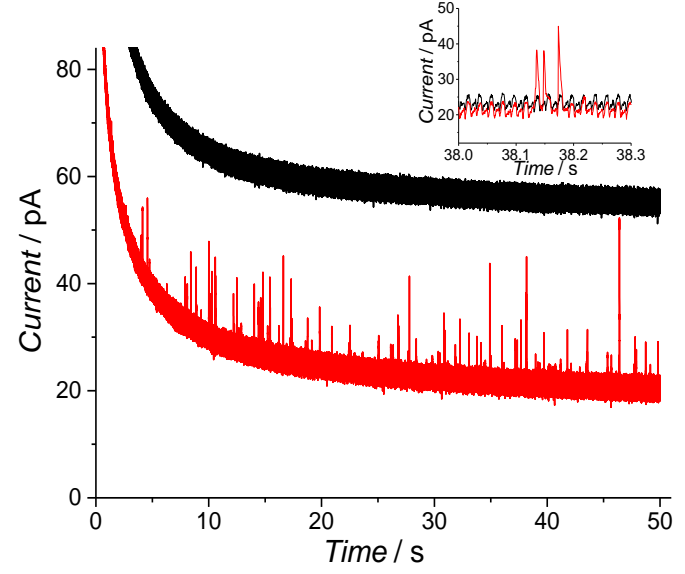

Figure 4. Fifty seconds chronoamperomogram for a carbon fibre microelectrode $(r=4.9 \mu \mathrm{m})$ immersed in $20 \mathrm{mM}$ sodium nitrate measured at + $0.6 \mathrm{~V}$ (vs MSE). Black: containing no nanoparticles. The black line is vertically shifted upwards by 33 pA for clarity. Red: containing 100 pM of PEG capped silver nanoparticles. Inset: A close up of individual signals observed.

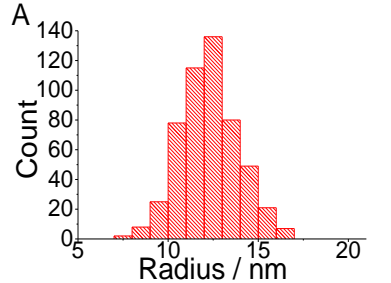

C

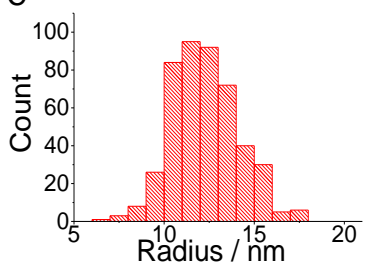

E

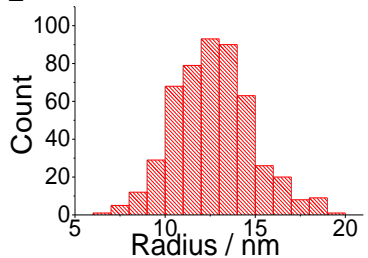

B

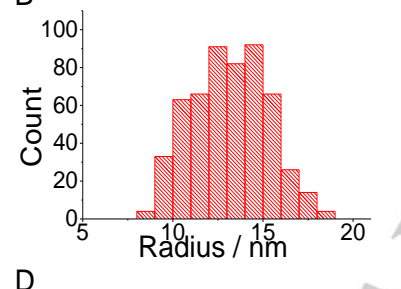

D

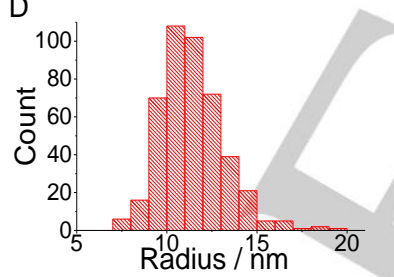

Table 1. Table showing the size of silver nanoparticles with different capping agent based on the two techniques of anodic particle coulometry and TEM.

\begin{tabular}{|c|c|c|c|c|}
\hline $\begin{array}{c}\text { Silver NP Capping } \\
\text { Agent }\end{array}$ & $\begin{array}{c}\text { Sizing from } \\
\text { Anodic } \\
\text { Particle } \\
\text { Coulometry } \\
(\mathrm{nm})\end{array}$ & $\begin{array}{l}\text { Number of } \\
\text { spikes } \\
\text { processed }\end{array}$ & $\begin{array}{l}\text { Number } \\
\text { of scans } \\
\text { recorded }\end{array}$ & $\begin{array}{l}\text { TEM } \\
(\mathrm{nm})\end{array}$ \\
\hline $\begin{array}{c}\text { Branched } \\
\text { Polyethylenimine } \\
\text { (BPEI) }\end{array}$ & $\begin{array}{c}12.3 \pm \\
1.6\end{array}$ & & 30 & $\begin{array}{c}15.0 \pm \\
1.6\end{array}$ \\
\hline Citrate & $\begin{array}{c}13.2 \pm \\
2.1\end{array}$ & 541 & 14 & $\begin{array}{c}14.6 \pm \\
2.1\end{array}$ \\
\hline Lipoic Acid & $\begin{array}{c}12.3 \pm \\
1.9\end{array}$ & 469 & 85 & $\begin{array}{c}13.5 \pm \\
3.9\end{array}$ \\
\hline $\begin{array}{l}\text { Polyethylene Glycol } \\
\text { (PEG) }\end{array}$ & $\begin{array}{c}11.4 \pm \\
1.7\end{array}$ & 448 & 27 & $\begin{array}{c}9.6 \pm \\
2.0\end{array}$ \\
\hline $\begin{array}{c}\text { Polyvinylpyrrolidone } \\
\text { (PVP) }\end{array}$ & $\begin{array}{c}12.7 \pm \\
2.2\end{array}$ & 500 & 39 & $\begin{array}{c}13.5 \pm \\
1.9\end{array}$ \\
\hline
\end{tabular}

nanoparticle has also shown a complete oxidation of the silver nanoparticles by matching the radius calculated from 'spikes' to the sizing by microscopy methods. ${ }^{[18 a, 18 c, 21]}$

Please note that Table 1 includes data relating to the impact frequency. These values are approximately consistent with an estimated frequency based on a simple Fickian diffusion model but the latter is known to overestimate the values because of electrode adsorption effects and also shielding of the micro disk electrode by adsorption on the insulting sheath. ${ }^{[22]}$ Also, the effects of hindered diffusion near surfaces can influence the data. ${ }^{[23]}$

\subsection{Influence of capping agents on oxidation}

In previous papers, it was reported that drop casting can cause nanoparticle aggregation, resulting in incomplete stripping during anodic stripping voltammetry. ${ }^{[14 a, 17]}$ Therefore, it is expected that the anodic stripping voltammetry performed by electrode modified via drop casting might result in an incomplete stripping. ${ }^{[17]}$ This results obtained in Figure 3 where the oxidative charges obtained from the five samples are lower than the expected charge of $26 \mu \mathrm{C}$ is consistent with the idea of incomplete stripping. In the earlier section, it was established that all five types of single nanoparticle, regardless of the capping agent, undergoes complete oxidation during nanoimpacts. Thus, comparing the electrochemical behaviour of single nanoparticle and drop casted nanoparticles, it is highly unlikely that the trend of partial oxidation in aggregates is caused by incomplete stripping of single nanoparticles. Therefore, the trend of partial oxidation observed in anodic particle coulometry is likely caused by the electrochemical behaviour of aggregates where some of the aggregates may entirely be inactivated or alternatively each aggregate may be partially oxidised..$^{[17]}$ 
This work presents the first time where silver nanoparticles capped with BPEI, lipoic acid, PEG and PVP are characterised by anodic particle coulometry. In a previous paper, platinum nanoparticles were capped with thiols of different alkyl chain length and it was found for chain length beyond 12 carbons, no signal was observed in the nano-imapct experiments. ${ }^{[24]}$ Hence, large capping agents such as 12-mercaptodececanic acid and 16-mercaptohexadecanoic acid have been reported to prevent the oxidation of the platinum nanoparticles. ${ }^{[24]}$ However, none of the capping agents in the current work prevent the complete oxidation of single nanoparticles in anodic particle coulometry. Therefore, it proves that the capping agents involved in these five samples are not large enough to block the redox reaction of the nanoparticle occurring on the electrode surface despite the large size of BPEI, PEG and PVP.

In anodic stripping voltammetry, the large difference in the partial oxidation of aggregates in Figure 2 suggests a significant influence of the capping agent on the behaviour of the aggregates. Thus, the different capping agents likely affect the partial oxidation of the nanoparticle aggregates to varying extents in anodic stripping voltammetry. This is crucially relevant to the use of anodic stripping voltammetry for nanoparticle quantification because most nanoparticles have capping agents on their surfaces. Therefore, careful analysis of anodic stripping voltammetry data is required when analysing nanoparticle aggregates with capping agent to avoid problems where the amount of nanoparticles on the surface may be severely underestimated by the oxidation signal.

\section{Conclusions}

In this work, the electrochemical techniques of anodic stripping voltammetry and anodic particle coulometry were performed on five differently capped silver nanoparticles to determine if there is a capping agent influence. From anodic particle coulometry, it was seen that the five capping agents considered do not affect the complete oxidation of the single silver nanoparticle. It is the first time where BPEI, lipoic acid, PEG and PVP capped silver nanoparticles are studied through anodic particle coulometry. Hence, it is viable to use the technique for nanoparticle detection and particle sizing in particular. In contrast with anodic stripping voltammetry, it is observed that capping agents strongly affect the size of the silver oxidation signal. It was found PEG capped silver nanoparticles give an oxidation signal of the largest magnitude among the five (the greatest percentage stripping was only 59\%) while PVP capped silver nanoparticles had the smallest stripping peak despite the same amount of silver deposited on the electrode surface. Therefore, it must be noted that the quantification of nanoparticles may be markedly underestimated in anodic stripping voltammetry because of capping agent effects.

\section{Experimental Section}

\section{Chemicals}

Five samples of silver nanoparticles with different capping agents were received from NanoComposix, San Diego, USA. The five samples used were branched polyethylenimine (BPEI) capped silver nanoparticle, citrate capped silver nanoparticle, lipoic acid capped silver nanoparticle, polyethylene glycol (PEG) capped silver nanoparticle and polyvinylpyrrolidone(PVP) capped silver nanoparticle. Sodium nitrate $\left(>99.5 \%, \mathrm{NaNO}_{3}\right)$ was obtained from Fisons Scientific Equipment, Loughborough, UK. Concentrated nitric acid $\left(>70 \%, \mathrm{H}_{2} \mathrm{SO}_{4}\right)$ and concentrated hydrochloric acid $(\sim 37 \%, \mathrm{HCl})$ were supplied by Fisher Scientific, Loughborough, UK. Ultrapure water from Millipore with resistivity not less than $18.2 \mathrm{M} \Omega . \mathrm{cm}$ at $25^{\circ} \mathrm{C}$ was used to prepare all solutions.

\section{Electrochemical Apparatus}

All electrochemical experiments were performed on a three electrode system in a Faraday cage. The electrochemical experiments were controlled by either a $\mu$ Autolab II or a PGSTAT302N from MetrohmAutolab BV, Utrecht, The Netherlands using the software of NOVA 1.10. A glassy carbon electrode of $3.0 \mathrm{~mm}$ diameter from $\mathrm{CH}$ instruments, Austin, USA was used as the working electrode for anodic stripping voltammetry. The glassy carbon electrode was polished to a mirror finish on diamond sprays from Kemet, Kent, UK in the size sequence of $3.0 \mu \mathrm{m}$, $1.0 \mu \mathrm{m}$ and $0.1 \mu \mathrm{m}$. For anodic particle coulometry, a carbon microdisc electrode of radius $4.9 \mu \mathrm{m}$ from BASi, West Lafayette, USA was used as the working electrode. The microelectrode was polished on alumina powder from Buehler, Coventry, UK in the size sequence of $1.0 \mu \mathrm{m}, 0.3$ $\mu \mathrm{m}$ and $0.05 \mu \mathrm{m}$ before experiments. A standard MSE (mercury/mercurous sulphate reference electrode $\left[\mathrm{Hg} / \mathrm{Hg}_{2} \mathrm{SO}_{4}, \mathrm{~K}_{2} \mathrm{SO}_{4}\right.$ (saturated)], $+0.62 \mathrm{~V}$ vs standard hydrogen electrode) was obtained from BASi, West Lafayette, USA. ${ }^{[25]}$ The counter electrode was a platinum mesh (99.99\%) from Goodfellow Cambridge Ltd, Huntingdon, UK. All electrochemical measurements were thermostated at $25 \pm 1{ }^{\circ} \mathrm{C}$.

\section{Nanoparticle characterisation}

Size characterisations were carried out using a JEOL 2010 analytical transmission electron microscope (TEM) (Herts, UK), which has a $\mathrm{LaB}_{6}$ electron gun and can be operated between 80 and 200kV. This instrument has a resolution of $0.19 \mathrm{~nm}$, an electron probe size down to $0.5 \mathrm{~nm}$ and a maximum specimen tilt of \pm 10 degrees along both axes. The instrument is equipped with an Oxford Instruments LZ5 windowless energy dispersive X-ray spectrometer (EDS) controlled by INCA software. It has facilities for point analysis as well as mapping and line scanning through the SemiStem controller. The TEM samples were prepared by depositing a drop of the silver nanoparticle suspension received on a carbon coated copper TEM grid and dried at room temperature for several hours before examination in the TEM. The silver nanoparticles capped by BPEl, citrate, lipoic acid, PEG and PVP were sized to have an average radius of $15.0 \pm 1.6 \mathrm{~nm}$ ( 30 particles), $14.6 \pm 2.1 \mathrm{~nm}$ (109 particles), $13.5 \pm 3.9 \mathrm{~nm}$ (18 particles), $9.6 \pm 2.0 \mathrm{~nm}$ (43 particles) and $13.5 \pm 1.9 \mathrm{~nm}$ (33 particles) respectively. Figure 6 shows the TEM images of the spherical silver nanoparticles. ImageJ software developed at the National Institutes of Health, USA was used to size the nanoparticles recorded on the TEM images. 

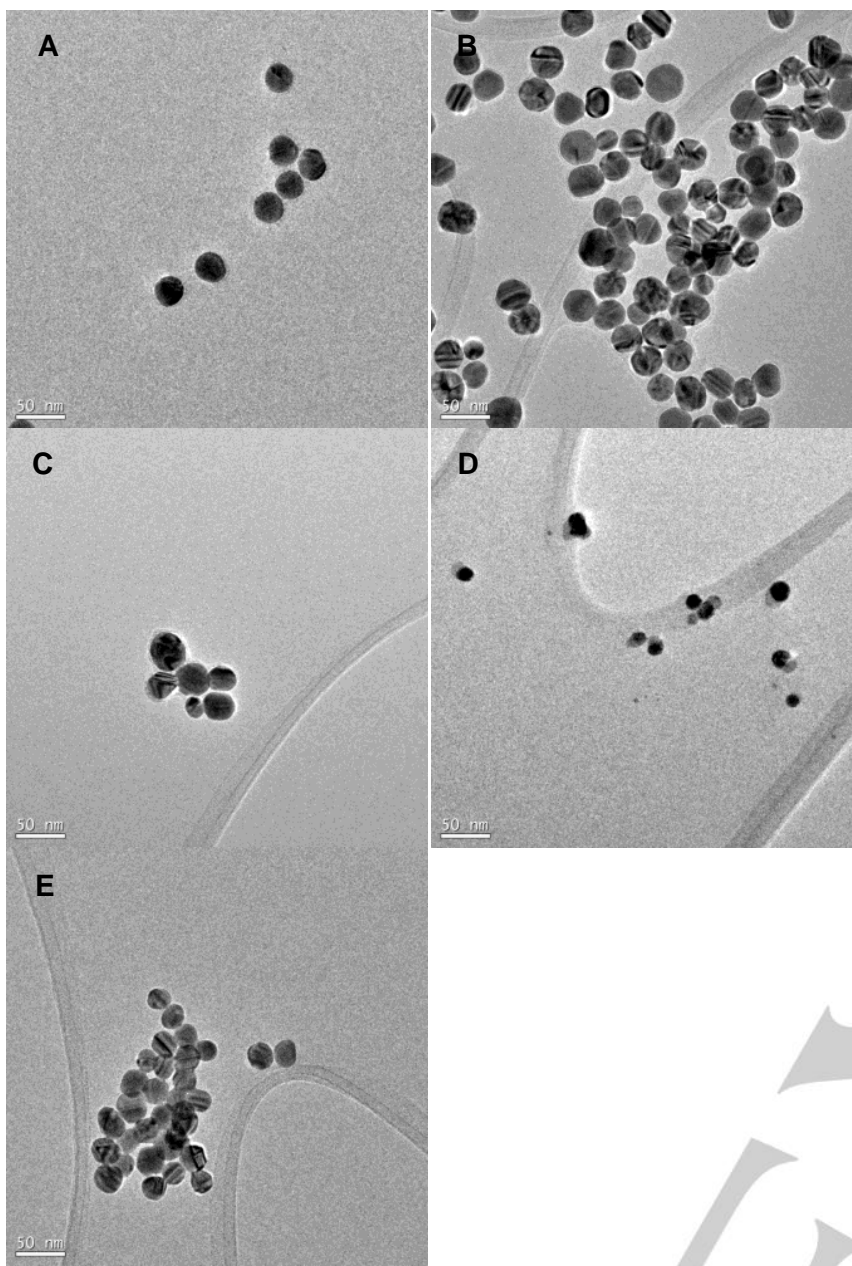

Figure 6. The TEM images of spherical silver nanoparticles with different capping agents. The scale bar is $50 \mathrm{~nm}$ in each picture. A: Branched Polyethylenimine (BPEI) capped silver nanoparticles; B: Citrate capped silver nanoparticles; C: Lipoic acid capped silver nanoparticles; D: Polyethylene Glycol (PEG) capped silver nanoparticles; E: Polyvinylpyrrolidone (PVP) capped silver nanoparticles.

Silver nanoparticle modified electrode for anodic stripping voltammetry

The silver nanoparticle suspension supplied was diluted by a factor of 2.23 with ultrapure water to ensure the total nanoparticle concentration was $100 \mathrm{pM}$. The working electrode was drop cast with $3 \mu \mathrm{L}$ of the diluted nanoparticle suspension and dried under a nitrogen flow. After drying, the nanoparticle modified electrode was immediately used for electrochemical experiments. A cyclic voltammogram was swept starting from $-0.6 \mathrm{~V}$ to $+0.5 \mathrm{~V}$ vs MSE at a scan rate of $50 \mathrm{mV} \mathrm{s}^{-1}$ to perform anodic stripping voltammetry.

\section{Anodic particle coulometry}

Prior to every anodic particle coulometry experiment, the electrochemical cell was soaked in aqua regia $\left(3 \mathrm{HCl}: 1 \mathrm{HNO}_{3}\right)$ for at least 30 minutes and sonicated in ultrapure water for 15 minutes to avoid any contamination by rogue nanoparticles. The silver nanoparticle suspension received was diluted with $20 \mathrm{mM}$ sodium nitrate solution to give an aliquot of $100 \mathrm{pM}$ of silver nanoparticles used for experiments. Chronoamperometric scans of fifty seconds duration with a sampling time of $0.0005 \mathrm{~s}$ were recorded at the potential of $+0.6 \mathrm{~V}$ vs MSE. The charge under each 'spike' was resolved by SignalCounter to determine the size of the nanoparticle detected. The software SignalCounter was developed by Dr. Dario Omanović from Division for Marine and Environmental Research, Ruđer Bošković Institutue, Zagreb, Croatia. ${ }^{[18 b, 19]}$

\section{Acknowledgements}

HST is supported by the National Research Foundation Singapore under its National Research Foundation (NRF) Environmental and Water Technologies (EWT) PhD Scholarship Programme and administered by the Environment and Water Industry Programme Office (EWI). The research leading to these results has received partial funding (RGC) from the European Research Council under the European Union's Seventh Framework Programme (FP/2007-2013)/ERC Grand Agreement n. [320403].

Keywords: silver $\cdot$ nanoparticles $\cdot$ aggregation $\bullet$ capping agent

[1] a) H. Bönnemann, Ryan M. Richards, Eur J Inorg Chem 2001, 2001 2455-2480; b) C. N. R. Rao, G. U. Kulkarni, P. J. Thomas, P. P Edwards, Chem Soc Rev 2000, 29, 27-35; c. Project on Emerging Nanotechnologies Analysis, http://www.nanotechproject.org/cpi/about lanalysis/ (Accessed 4 Feb 2014). d) W. Z. Teo, M. Pumera, RSC Adv 2014, 4, 5006.

[2] M. Vert, Y. Doi, K.-H. Hellwich, M. Hess, P. Hodge, P. Kubisa, M. Rinaudo, F. Schué, Pure Appl Chem 2012, 84, 377-410.

[3] W. J. Plieth, J Phys Chem 1982, 86, 3166-3170.

[4] a) K. D. Kim, D. N. Han, H. T. Kim, Chem Eng J 2004, 104, 55-61; b) B. Dobias, X. Qiu, W. V. Rybinski, Solid-Liquid Dispersions, Vol. 81 Mercel Dekker Inc., New York, 1999, pp. 127,255.

[5] A. M. El Badawy, K. G. Scheckel, M. Suidan, T. Tolaymat, Sci Total Environ 2012, 429, 325-331.

[6] P. Raveendran, J. Fu, S. L. Wallen, J Amer Chem Soc 2003, 125 13940-13941.

[7] C. Luo, Y. Zhang, X. Zeng, Y. Zeng, Y. Wang, J Colloid Interface Sci 2005, 288, 444-448.

[8] a) Z. S. Pillai, P. V. Kamat, J Phys Chem B 2004, 108, 945-951; b) A Pyatenko, M. Yamaguchi, M. Suzuki, J Phys Chem B 2005, 109, 21608-21611.

[9] a) S. Bhattacharya, A. Srivastava, A. Pal, Angew Chem 2006, 118, 3000-3003; b) J. N. Kuhn, C.-K. Tsung, W. Huang, G. A. Somorjai, J Catal 2009, 265, 209-215; c) L. Kvítek, A. Panáček, J. Soukupová, M. Kolář, R. Večeřová, R. Prucek, M. Holecová, R. Zbořil, J Phys Chem C 2008, 112, 5825-5834.

[10] H. S. Toh, C. Batchelor-McAuley, K. Tschulik, R. G. Compton, Science China Chemistry 2014, 57, 1199-1210.

[11] a) D. D. Evanoff, Jr., G. Chumanov, ChemPhysChem 2005, 6, 1221 1231; b) D. D. Evanoff, Jr., G. Chumanov, J Phys Chem B 2004, 108, 13957-13962; c) W. Haiss, N. T. K. Thanh, J. Aveyard, D. G. Fernig, Anal Chem 2007, 79, 4215-4221; d) M. S. Abd El-sadek, A. Y. Nooralden, S. Moorthy Babu, P. K. Palanisamy, Opt Comm 2011, 284, 2900-2904.

[12] a) M. K. Temgire, S. S. Joshi, Radiat Phys Chem 2004, 71, 1039-1044; b) M. Stevanović, B. Kovačević, J. Petković, M. Filipič, D. Uskoković, Int J Nanomed 2011, 6, 2837-2847. 
[13] a) J. Wang, Analytical Electrochemistry, 3rd ed., John Wiley \& Sons, Inc., Hoboken, New Jersey, 2006; b) R. G. Compton, C. E. Banks, Understanding Voltammetry, 2nd ed., World Scientific, Singapore, 2011.

[14] a) H. S. Toh, C. Batchelor-McAuley, K. Tschulik, M. Uhlemann, A. Crossley, R. G. Compton, Nanoscale 2013, 5, 4884-4893; b) C. C. M Neumann, C. Batchelor-McAuley, K. Tschulik, H. S. Toh, P. Shumbula, J. Pillay, R. Tshikhudo, R. G. Compton, ChemElectroChem 2014, 1, 87 89; c) K. Tschulik, C. Batchelor-McAuley, H. S. Toh, E. J. Stuart, R. G. Compton, Phys Chem Chem Phys 2014, 16, 616-623; d) M. Pumera, M. Aldavert, C. Mills, A. Merkoçi, S. Alegret, Electrochim Acta 2005, 50 3702-3707; e) Y. Wang, E. Laborda, B. J. Plowman, K. Tschulik, K. R. Ward, R. G. Palgrave, C. Damm, R. G. Compton, Phys Chem Chem Phys 2014, 16, 3200-3208; f) X. F. Zhou, W. Cheng, C. BatchelorMcauley, K. Tschulik, R. G. Compton, Electroanalysis 2014, 26, 248253.

[15] M. Mannini, Molecular Magnetic Materials On Solid Surfaces, Firenze University Press, Firenze, 2008.

[16] IUPAC. Compendium of Chemical Terminology, 2nd ed. (the "Gold Book"). Compiled by A. D. McNaught and A. Wilkinson. Blackwell Scientific Publications, Oxford, 1997.

[17] S. J. Cloake, H. S. Toh, P. T. Lee, C. Salter, C. Johnston, R. G. Compton, ChemistryOpen 2014, DOI: 10.1002/open.201402050.

[18] a) Y.-G. Zhou, N. V. Rees, R. G. Compton, Angew Chem Int Ed 2011, 50, 4219-4221; b) E. J. E. Stuart, K. Tschulik, D. Omanovic, J. T. Cullen, K. Jurkschat, A. Crossley, R. G. Compton, Nanotechnology 2013, 24,
444002; c) E. J. E. Stuart, N. V. Rees, J. T. Cullen, R. G. Compton, Nanoscale 2013, 5, 174-177; d) E. J. E. Stuart, K. Tschulik, C Batchelor-McAuley, R. G. Compton, ACS Nano 2014.

[19] J. Ellison, K. Tschulik, E. J. Stuart, K. Jurkschat, D. Omanović, M. Uhlemann, A. Crossley, R. G. Compton, ChemistryOpen 2013, 2, 69-75.

[20] H. S. Toh, C. Batchelor-McAuley, K. Tschulik, R. G. Compton, Analyst 2013, 138, 4292-4297.

[21] E. J. E. Stuart, K. Tschulik, D. Omanović, J. T. Cullen, K. Jurkschat, A. Crossley, R. G. Compton, Nanotechnology 2013, 24, 444002.

[22] a) E. J. E. Stuart, Y.-G. Zhou, N. V. Rees, R. G. Compton, RSC Adv 2012, 2, 6879-6884; b) E. Kätelhön, W. Cheng, C. Batchelor-McAuley, K. Tschulik, R. G. Compton, ChemElectroChem 2014; c) E. Katelhon, R. G. Compton, Analyst 2014, 139, 2411-2415; d) S. Eloul, R. G. Compton, ChemElectroChem 2014, 1, 917-924.

[23] E. Kätelhön, R. G. Compton, Chem. Sci. 2014, 5, 4592-4598.

[24] X. Xiao, S. Pan, J. S. Jang, F.-R. F. Fan, A. J. Bard, J Phys Chem C 2009, 113, 14978-14982

[25] CRC Handbook of Chemistry and Physics 2012-2013, ed. W. M. Haynes, CRC Press, Florida, 93rd edn., 2012.. 


\section{Entry for the Table of Contents}

\section{FULL PAPER}

The influence of capping agents on the oxidation of silver nanoparticles is studied through anodic stripping voltammetry and 'nano-impacts'. The former is unreliable when the nanoparticles are aggregated whereas the latter is quantitative.

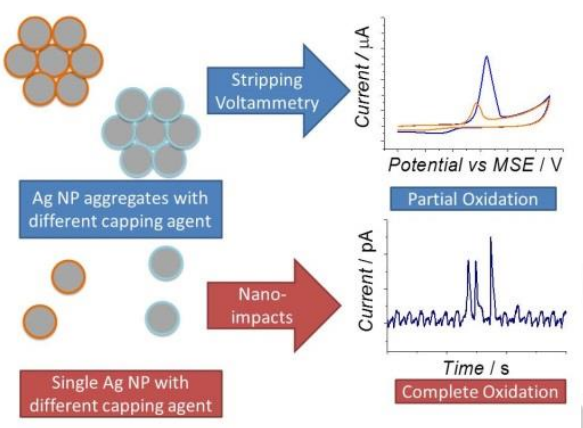

H.S. Toh, K. Jurkschat, and R.G. Compton*

Page No. - Page No.

The Influence of the Capping Agent on the Oxidation of Silver Nanoparticles: Nano-impacts vs Stripping Voltammetry 